\title{
THREE-DIMENSIONAL CALCULATION OF SINUS AUGMENTATION VOLUME AFTER MAXILLARY SINUS FLOOR ELEVATION
}

\begin{abstract}
Objective: This study aimed to calculate the volume of the maxillary sinus and grafted part of it with different modes of three-dimensional reconstruction software.
\end{abstract}

Materials and Methods: This retrospective volumetric cone-beam computed tomography study was carried out on 21 patients/ 36 maxillary sinuses who had undergone maxillary sinus lift surgery using a lateral approach, between 2010 and 2016 at the Department Periodontology. All statistical analyses were performed by using the NCSS (Number Cruncher Statistical System, Kaysville, Utah, U.S.A.) software. $\mathrm{P}$ values $<0.05$ were considered to indicate statistical significance.

Results: The mean volume of the right maxillary sinus cavity was 15.37 $\mathrm{cm}^{3}$ while for the left it was $15.90 \mathrm{~cm}^{3}$. There was no statistically significant difference between right and left maxillary sinus volumes ( $p>0.05$ ). An occupied portion of the sinus cavity after lateral sinus floor elevation surgery was approximately $14.87 \%$. Furthermore, the volume through manual and automatical modes of the software do not affect the results ( $p>0.05)$. The right and left sides of the maxillary sinus volumes were not different from each other. Gender and measuring mode was not found to be related to volumetric values of the maxillary sinus cavity.

Conclusions: The grafted volume of the sinus cavity was defined as a safe volume in terms of serious complications during and after the surgery.

Key words: Sinus floor elevation, maxillary sinus volume, cone-beam computed tomography, dental implant, Schneiderian membrane.

\author{
*Samir Goyushov ${ }^{1}$ \\ Erhan Dursun ${ }^{2}$ \\ Kaan Orhan ${ }^{3}$ \\ Tolga F. Tözüm ${ }^{4}$
}

\footnotetext{
${ }^{1}$ Department of Periodontology, Faculty of Dentistry, Istanbul Aydin University, Istanbul, Turkey.

${ }^{2}$ Department of Periodontology, Faculty of Dentistry, Hacettepe University, Ankara, Turkey.

${ }^{3}$ Department of Dentomaxillofacial Radiology, Faculty of Dentistry, Ankara University, Ankara, Turkey.

${ }^{4}$ Department of Periodontics, College of Dentistry, University of Illinois at Chicago, Chicago, IL, U.S.A.
}

How to Cite: Goyushov S, Dursun E, Orhan K, Tözüm TF. Three-Dimensional Calculation of Sinus Augmentation Volume after Maxillary Sinus Floor Elevation. Cumhuriyet Dent J 2021;24:2:163-169.

*Corresponding Author:

Department of Periodontology, Faculty of Dentistry, Istanbul Aydin University, Istanbul, Turkey.

Phone: +905413417183r E-mail: samir.goyush@gmail.com 


\section{INTRODUCTION}

The maxillary sinus is the largest of the paranasal sinus with pyramid-shaped cavity between the nasal wall and zygoma. ${ }^{1}$ The cavity extends from premolar area to the first molar region, and average volume of it is approximately 12 to $15 \mathrm{~cm} 33^{2,3}$ The pneumatization of the maxillary sinus varies from patient to patient, and increases with age in the edentulous areas towards the alveolar process. ${ }^{4}$

Alveolar bone resorption and sinus pneumatization can compromise immediate implantation in the posterior maxilla. ${ }^{5}$ Different sinus floor elevation techniques have been used since 1980 to restore this anatomic deficiency before the implant surgery. ${ }^{6,7}$ Among these techniques, lateral sinus floor elevation (LSFE) has been recommended in sites with insufficient residual bone height $(\mathrm{RBH}){ }^{8}$ LSFE ensured the reconstruction of bone deficiencies of the sinus cavities with high clinical success rates, allowing implantation in that region safer and more predictable. $^{9}$

Moreover, volumetric knowledge of the maxillary sinus cavity and grafted part of it is crucial for the practitioners in choosing the surgical method and evaluate the success of the procedure. The various effects of LSFE on maxillary sinus physiology were evaluated in this retrospective study. Maxillary sinus physiology might also be affected by the volumetric changes after sinus surgeries. It has been established that the risk of Schneiderian membrane perforations is greatly correlated to the membrane thickness. ${ }^{10,11}$ One of research objectives was to analyze the change of thickness of the Schneiderian membrane and gingival thickness before and after LSFE.

Several radiological and clinical studies have reported promising data through panoramic radiography, spiral computerized tomography (CT), cone-beam computed tomography (CBCT), magnetic resonance imaging (MRI) to provide volumetric information of the sinus cavity. ${ }^{12-15}$ Among these methods, the application of CBCT in exploring paranasal sinuses has rapidly become a popular tool by supporting scientific and technologic advances. ${ }^{16}$ CBCT was accepted very efficient, affordable, and relatively safe method for evaluating maxillary sinus cavity. One of the main points of this study to define a safe volume of the sinus cavity to avoid any serious complications during the surgery. The present radiographic study observed total volume of the sinus cavity and the grafted part of the cavity after LSFE procedure. Another aim of the study is to compare the volumetric values obtained by manual and automatic volumetric measurements.

\section{MATERIAL AND METHODS \\ Patient selection}

The study included the radiographic data of 21 patients, 36 maxillary sinuses. During the period from 2010 to 2016, these patients had undergone CBCT investigations before maxillary sinus lift (MSL) using bone biomaterials at the Department of Periodontology. Fifteen patients (71.4\%) underwent bilateral LSFE surgery. LSFE surgeries were carried out at the same department. According to the patients' records, in all sites, pre-surgical bone height was lower than $5 \mathrm{~mm}$ and a staged surgical approach was necessary. ${ }^{17,18}$ Thirty-six maxillary sinuses which underwent maxillary sinus lifting surgeries with the application of bone substitutes were defined as research subjects. Collagenated heterologous bone graft derived (Large granules, 12mm, Apatos, Osteobiol ${ }^{\circledR}$, Tecnoss, Coazze, Italy) was used to fill the antrum. The length of the paced implants into the augmented sinuses were between $10-12 \mathrm{~mm}$. No perforation of the sinus membrane or any other clinical complications such as postoperative sinusitis, ostium obstruction were present in the patient records. The study did not address patient's general health condition at the time of examination and surgery, as well as the elapsed time between tooth loss, MSL and the smoking factor. The study was approved by the Local Research Ethics Committee of YYY (Decision date: 07/11/2017, decision number: 1511), and the protocol of the study was conducted following the principles of the Declaration of Helsinki.

\section{Imaging procedures}

Radiological investigation for pre-surgical planning and post-surgical evaluation purposes were performed using CBCT device I-CAT Next Generation (Imaging Sciences International, Hatfield, PA, U.S.A.). All patients were investigated following a unified protocol. All 
included patients' second radiographs were taken because of different diagnostic purposes other than the sinus surgery. Radiographic records of the patients showed the same scanning parameters: diameter $-16 \mathrm{~cm}$, height $-13 \mathrm{~cm}$, scanning time 8 to 9 seconds, power $-120 \mathrm{kV}, 5 \mathrm{~mA}$. Images were obtained using 0.3 voxel (three-dimensional image volume unit) size. Images were processed and reconstructed by Three Dimensional (3D) Synapse Software (Fujifilm, Tokio, Japan). Before taking measurements, an image was positioned so that the plane of the hard palate was parallel to the floor while the sagittal plane was perpendicular to the floor. Each volumetric data was measured twice (in automatic (Fig. 1) and in manual mode (Fig. 2) and recorded in cubic centimeters $\left(\mathrm{cm}^{3}\right)$.

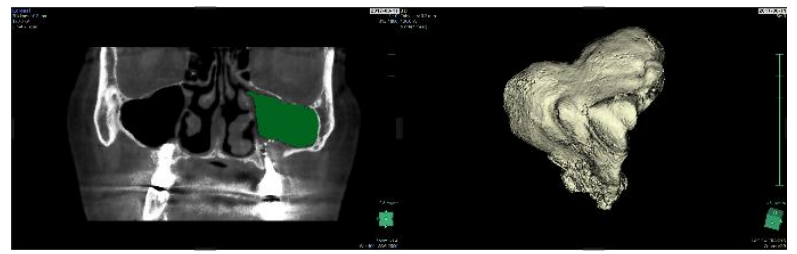

Figure 1. Total sinus volume measurement and visual construction of the cavity (automatically).

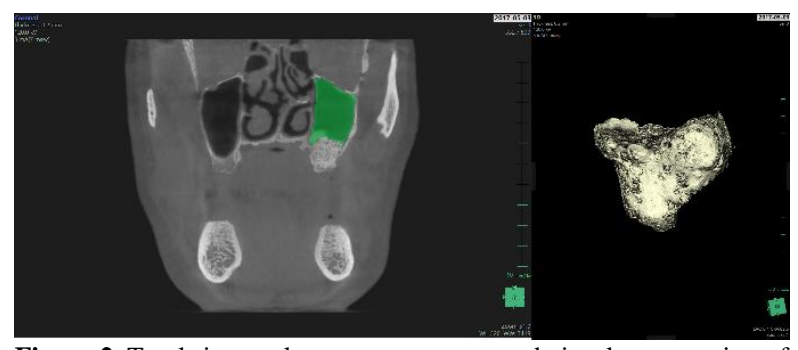

Figure 2. Total sinus volume measurement and visual construction of the cavity (manually).

\section{Statistical analysis}

The recorded data were processed using NCSS software (Number Cruncher Statistical System, Kaysville, Utah, U.S.A.). After descriptive and comparative analysis, significance of $5 \%$ or less Table 1. Volumetric measurements of the sinus cavities.

\begin{tabular}{ccccc}
\hline Measurement Mode & Side & $\begin{array}{c}\text { Volume }\left(\mathbf{c m}^{\mathbf{3}}\right) \\
\pm \text { SD }\end{array}$ & $\begin{array}{c}\text { Difference } \\
\text { (p value) }\end{array}$ & $\begin{array}{c}\text { Difference } \\
\text { (p value) }\end{array}$ \\
\hline \multirow{2}{*}{ Manual } & Right & $15.37( \pm 3.05)$ & & \\
& Left & $15.9( \pm 3.08)$ & $\mathrm{p}>0.05$ & \\
& Mean & $15.35( \pm 2.9)$ & & \\
& Right & $15.19( \pm 3.08)$ & $\mathrm{p}>0.05$ \\
Automatic & Left & $14.98( \pm 2.87)$ & & \\
& Mean & $15.1( \pm 2.9)$ & & \\
& & &
\end{tabular}

was considered statistically significant. When the data were subjected to normal distribution, analysis of variance (ANOVA) was used for analysis of quantitative differences between two or more groups Student's t-test was performed for analysis of differences between two groups.

\section{RESULTS}

Summarization of demographic data led to the conclusion that out of 36 maxillary sinuses included in the study $22(61.1 \%)$ were females and 14 (38.9\%) were males. At the time of LSFE surgery, the mean age of patients was 50.9 years (31-66 years). Mean age of females who were included in the study was 49.4 while the mean age of males was 53.7 years.

Inclusion criteria consisted of maxillary CBCT taken both before and after the sinus surgery. The longest time recorded from postsurgery until CBCT re-examination was 5 years. The average time from post-surgery until CBCT re-examination was $2.06 \pm 0.749$ years.

Mean values and standard deviations of the volumes for the maxillary sinus cavities and for grafts are shown in Table 1 and Table 2. All volumetric assessments were performed not only automatically but also in manual mode using 3D Synapse Software. Although there were small differences between manual and automatic measurements, they were not significant ( $p>0.05)$. The total maxillary sinus volume (mean $\pm \mathrm{SD}$ ) measured manually on CBCT images was $15.35 \pm$ $2.9 \mathrm{~cm}^{3}$. The minimum maxillary sinus volume was $10.91 \mathrm{~cm}^{3}$ and the maximum was $22.03 \mathrm{~cm}^{3}$. 
Table 2. Volumetric measurements of the grafts.

\begin{tabular}{cccc}
\hline Measurement Mode & $\begin{array}{c}\text { Volume }\left(\mathbf{c m}^{3}\right) \\
\pm \text { SD }\end{array}$ & Percentage & $\begin{array}{c}\text { Difference } \\
(\mathbf{p} \text { value) }\end{array}$ \\
\hline Manual & $2.25( \pm 1.24)$ & $14.87 \%( \pm 7.71)$ & $\mathrm{p}>0.05$ \\
Automatic & $2.24( \pm 1.2)$ & $14.66 \%( \pm 7.73)$ & \\
\hline
\end{tabular}

The overall average graft volume obtained after the surgery was $2.25 \mathrm{~cm}^{3}\left( \pm 1.24 \mathrm{~cm}^{3}\right)$. The occupied space of the sinus cavity by graft biomaterials was calculated (\%), as shown in Table 2 . The average percentage of the grafted part was $14.87 \%$ through manual measurements and $14.66 \%$ through automatic measurements.

In pre-surgical CBCT imaging, the mean thickness of the crestal gingiva was $1.09 \mathrm{~mm}$
$( \pm 0.94)$. The gingival thickness in the surgical area increased slightly after the surgery, which was not statistically significant (Table 3 ). The sinus membrane thickness ranged from $0.7 \mathrm{~mm}$ to $3.2 \mathrm{~mm}$ in pre-surgical CBCT scans. The mean thickness prior to surgery was $1.62 \mathrm{~mm}$. Following LSFE, mean membrane thickness showed no significant difference from pre-surgical values (Table 3 ).

Table 3. Analysis of the linear measurements before and after sinus surgery

\begin{tabular}{cccc}
\hline Parameters & $\begin{array}{c}\text { Pre-op. }(\mathbf{m m}) \\
\pm \text { SD }\end{array}$ & $\begin{array}{c}\text { Post-op. }(\mathbf{m m}) \\
\pm \text { SD }\end{array}$ & $\begin{array}{c}\text { Difference } \\
\text { (p value) }\end{array}$ \\
\hline Schneiderian membrane thickness & $1.62( \pm 1.04)$ & $1.77( \pm 0.72)$ & $\mathrm{p}>0.05$ \\
Gingival thickness & $1.09( \pm 0.94)$ & $1.29( \pm 0.47)$ & $\mathrm{p}>0.05$ \\
Residual bone height & $3.14( \pm 2.38)$ & --- & --- \\
Graft height & --- & $11.36( \pm 2.78)$ & --- \\
\hline
\end{tabular}

All ostiums were detected and most of them maintained their openness after sinus lift surgery. Obstruction of the sinus ostium was observed just in one sinus $(2.7 \%)$. The mean buccal bony wall of the maxillary sinus cavity after the surgery was slightly thicker than in pre-surgical CBCT scans (Table 3).

\section{DISCUSSION}

According to several clinical studies, the success rate of the sinus elevation procedure for the placement of implants has significantly increased. ${ }^{16}$ LSFE with different types of bone grafts has become a remarkable treatment choice for severely atrophic posterior maxilla. This retrospective analysis aimed to evaluate the physiological changes after sinus lifting surgery.

Volumetric and linear measurements of the maxillary sinus cavity have conventionally been measured using cadavers by different authors. ${ }^{2,19,20}$ However, "gold standard" for LSFE considered 3D CBCT. $^{21}$ Ariji, Uchida and others have measured maxillary sinus volume in humans through the CT images., ${ }^{4,22,23}$ Recent years, volumetric measurements have been performed by using CBCT with different modelling software. $^{16,24,25}$ In this study, total and grafted volume of the maxillary sinus were examined using manual and automatic mode of the 3D Synapse Software.

The maxillary sinus cavity is described as the largest one between paranasal sinuses and its volume is changing between $8.6 \mathrm{~cm}^{3}$ and 24.9 $\mathrm{cm}^{3} .3,26$ The maxillary sinus volume tends to be increased after the maxillary posterior tooth loss. ${ }^{27}$ According to Takahashi et al. ${ }^{28}$ mean volume of the maxillary sinus was $31.3 \mathrm{~cm}^{3}$, which was approximately twice as much as the present results. This difference may be explained by the fact that volume measuring methods were totally unlike from each other. The mean volume of the sinus cavity after full growth was calculated as 14.8 $\mathrm{cm}^{3}{ }^{29}$ The mean total sinus volume was around 15 $\mathrm{cm}^{3}$ in the present results.

There have been a few studies reported graft volume after different types of sinus surgeries..$^{30-33}$ 
However, this is the first CBCT study in the literature using the current software to investigating the safe volume of the sinus cavity for the grafting. The safe volume after sinus grafting means not to interfere with the osteomeatal unit that hazardous to sinus physiology. In this study, all included patients were performed successfully two-stage lateral sinus floor elevation without any complication. These results confirmed that sinus augmentation with lateral approach is a safe procedure when evaluation of maxillary sinus anatomy by CBCT and 3D elevation of the membrane are performed.

All volumetric measurements of the sinuses were performed twice to compare manual and automatic modes of the software. To the best of our knowledge, no study evaluated the difference between manual and automatical estimated maxillary sinus cavity volumes in the CBCT images. The automatical volume calculation is easier to perform and less time consuming than the manual measurement. Our study showed an excellent convenience between the manual and automatic measuring volumetric data of the sinus cavity. Although such estimation may not be suitable for other software, we believe that this tool might be beneficial for research purposes in further evaluations through this program.

Published studies comparing left and right sinus cavity volumes have produced resemble outcomes. Most of the authors have found no statistical difference between right and left cavity in terms of sinus volume. ${ }^{4,34}$ Our results are consistent with other reported studies. In the present study, the mean value of the right sinus cavity volume was $15.37 \mathrm{~cm}^{3}$, and $15.9 \mathrm{~cm}^{3}$ for the left side, which showed no statistically significant difference.

In the present study, the authors relied on CBCT exploration in order to evaluate and understand physiological changes after LSFE. Sinus membrane thickness is one of the first postsurgical inflammatory signs inside the maxillary sinus cavity after membrane elevation procedure. The transient swelling of the Schneiderian membrane after the LSFE has already been described by Quirynen et $a l^{35}$ Membrane thickening was assessed before and after the sinus surgery to understand the effect of the procedure on membrane physiology. The outcomes showed the fully resolution of the early post-surgical inflammatory reaction of sinus membrane after the healing period. These presented results are in excellent agreement with the previous established results by different authors. ${ }^{3637}$

Some authors reported that healthy Schneiderian membranes are thicker in individuals with a thick gingival biotype than in those with a thin gingival biotype. ${ }^{11,38}$ However, our results showed no correlation between the membrane thickness and crestal gingival thickness.

Sample size might be a major limitation of this retrospective study. Moreover, the current study may be considered as standardized since they were only augmented with bone grafts without implant placement. Further studies can be designed to achieve valuable data about graft volumes used with different surgical techniques.

\section{CONCLUSIONS}

The present study showed that in posterior maxillary regions with insufficient bone height, the LSFE technique could achieve a predictable outcome for implant surgery. According to the results of this retrospective study, approximately $14.87 \%$ of the sinus cavity is occupying by graft materials after sinus elevation surgery, which considered as a safe volume. There were no significant difference between pre-surgical and post-surgical CBCT scans according to sinus membrane thickness and patency of osteomeatal unit. Nevertheless, this retrospective radiographic study confirmed that sinus augmentation surgery is a safe procedure in a long term when carefully planned and all inflammatory reactions totally subsides after the healing period.

\section{REFERENCES}

1. Lerno P. Identification using the maxillary sinus. Chir Dent Fr. 1983; 53: 39-40.

2. Uchida Y, Goto M, Katsuki T, Akiyoshi T. A cadaveric study of maxillary sinus size as an aid in bone grafting of the maxillary sinus floor. J Oral Maxillofac Surg. 1998; 56: 1158-1163. 
3. Anagnostopoulou S, Venieratos D, Spyropoulos N. Classification of human maxillar sinuses according to their geometric features. Anat Anz. 1991; 173: 121-130.

4. Ariji Y, Kuroki T, Moriguchi S, Ariji E, Kanda S. Age changes in the volume of the human maxillary sinus: a study using computed tomography. Dentomaxillofac Radiol. 1994; 23: 163-168.

5. Tatum JH. Maxillary and sinus implant reconstructions. Dent Clin North Am. 1986; 30: 207229.

6. Lopez MA, Andreasi MB, Confalone L, Lico S, Carinci F. Crestal sinus lift using a fluido-dynamic computer guided precise and atraumatic technique. J Biol Regul Homeost agents. 2015; 29: 67-73.

7. Zitzmann NU, Schärer P. Sinus elevation procedures in the resorbed posterior maxilla: Comparison of the crestal and lateral approaches. Oral Surg Oral Med Oral Pathol Oral Radiol Endod. 1998; 85: 8-17.

8. Jensen OT, Shulman LB, Block MS, Iacono VJ. Report of the sinus consensus conference of 1996. Int J Oral Maxillofac Implants. 1998; 13: 11-45.

9. Del Fabbro M, Testori T, Francetti L, Weinstein R. Systematic review of survival rates for implants placed in the grafted maxillary sinus. Int $\mathbf{J}$ Periodontics Restorative Dent. 2004; 24: 565-577.

10. Lin YH, Yang YC, Wen SC, Wang HL. The influence of sinus membrane thickness upon membrane perforation during lateral window sinus augmentation. Clin Oral Implants Res. 2016; 27: 612-617.

11. Aimetti M, Massei G, Morra M, Cardesi E, Romano F. Correlation between gingival phenotype and Schneiderian membrane thickness. Int Journal Oral Maxillofac Implants. 2008; 23: 1128-1132.

12. Aranyarachkul $P$, Caruso J, Gantes B, Schulz E, Riggs M, Dus I, Yamada JM, Crigger M. Bone density assessments of dental implant sites: 2. Quantitative cone-beam computerized tomography. Int $\mathrm{J}$ Oral Maxillofac Implants. 2005; 20: 416-424.

13. Hallman M, Hedin M, Sennerby L, Lundgren S. A prospective 1-year clinical and radiographic study of implants placed after maxillary sinus floor augmentation with bovine hydroxyapatite and autogenous bone. J Oral Maxillofac Surg. 2002; 60: 277-284.
14. Valentini P, Abensur D. Maxillary sinus floor elevation for implant placement with demineralized freeze-dried bone and bovine bone (Bio-Oss): a clinical study of 20 patients. Int J Periodont Rest Dent. 1997; 17: 232-241.

15. Quirynen M, Lamoral Y, Dekeyser C, Peene P, Steenberghe VD, Bonte J, Baert AL. The CT scan standard reconstruction technique for reliable jaw bone volume determination. Int J Oral Maxillofac Implants. 1990; 5: 36-41

16. Gulec M, Tassoker M, Magat G, Lale B, Ozcan S, Orhan K. Three-dimensional volumetric analysis of the maxillary sinus: a cone-beam computed tomography study. Folia Morphol. 2019.

17. K, Nilsson P, Hirsch JM. Sinus lifting procedure: I. One-stage surgery with bone transplant and implants. Clin Oral Implants Res. 2001; 12: 479-487.

18. Wang H-L, Katranji A. ABC sinus augmentation classification. Int J Periodont Rest Dent. 2008; 28; 383389.

19. Kraut RA. A cadaveric study of maxillary sinus size as an aid in bone grafting of the maxillary sinus floor. $\mathrm{J}$ Oral Maxillofac Surg. 1998; 56: 1163.

20. Schaeffer JP. The sinus maxillaris and its relations in the embryo, child, and adult man. Am J Anat. 1910; 10: 313-368.

21. Stutzki M, Jahns E, Mandapathil MM, Diogo I, Werner JA, Güldner C. Indications of cone beam CT in head and neck imaging. Acta oto-laryngol. 2015;135:1337-1343.

22. Uchida Y, Goto M, Katsuki T, Soejima Y. Measurement of maxillary sinus volume using computerized tomographic images. Int $\mathbf{J}$ Oral Maxillofac Implants. 1998; 13: 811-818.

23. Emirzeoglu M, Sahin B, Bilgic S, Celebi M, Uzun A. Volumetric evaluation of the paranasal sinuses in normal subjects using computer tomography images: a stereological study. Auris Nasus Larynx. 2007; 34: 191195.

24. Belgin CA, Colak M, Adiguzel O, Akkus Z, Orhan $\mathrm{K}$. Three-dimensional evaluation of maxillary sinus volume in different age and sex groups using CBCT. Eur Arch Otorhinolaryngol. 2019; 276(5): 1493-1499. 
25. Dellavia C, Speroni S, Pellegrini G, Gatto A, Maiorana C. A new method to evaluate volumetric changes in sinus augmentation procedure. Clin Implant Dent Relat Res. 2014; 16: 684-690.

26. Lawson W, Patel ZM, Lin FY. The development and pathologic processes that influence maxillary sinus pneumatization. Anat Rec: Advances in Integrative Anatomy and Evolutionary Biology: Advances in Integrative Anatomy and Evolutionary Biology. 2008; 291: 1554-1563.

27. Farina R, Pramstraller M, Franceschetti G, Pramstraller C, Trombelli L. Alveolar ridge dimensions in maxillary posterior sextants: a retrospective comparative study of dentate and edentulous sites using computerized tomography data. Clin Oral Implants Res. 2011; 22: 1138-1144.

28. Takahashi Y, Watanabe T, Iimura A, Takahashi O. A study of the maxillary sinus volume in elderly persons using Japanese cadavers. Okajimas Folia Anat Japonica. 2016; 93: 21-27.

29. Park I-H, Song JS, Choi H, Kim TH, Hoon S, Lee $\mathrm{SH}$, Lee HM. Volumetric study in the development of paranasal sinuses by CT imaging in Asian: a pilot study. Int J Pediatr Otorhinolaryngol. 2010; 74: 1347-1350.

30. Kwon JJ, Hwang J, Kim YD, Shin SH, Cho BH, Lee JY. Automatic three-dimensional analysis of bone volume and quality change after maxillary sinus augmentation. Clin Implant Dent Relat Res. 2019; 21: 1148-1155.

31. Sonoda T, Harada T, Yamamichi N, Monje A, Wang HL. Association Between Bone Graft Volume and Maxillary Sinus Membrane Elevation Height. Int J Oral Maxillofac Implants. 2017; 32: 735-740.
32. Nagla'a A, Bahammam MA. Cone Beam CT-Based Preoperative Volumetric Estimation of Bone Graft Required for Lateral Window Sinus Augmentation, Compared with Intraoperative Findings: A Pilot Study. Open Dent J. 2018; 12: 820-826.

33. Alayan J, Ivanovski S. A prospective controlled trial comparing xenograft/autogenous bone and collagenstabilized xenograft for maxillary sinus augmentationComplications, patient-reported outcomes and volumetric analysis. Clin Oral Implants Res. 2018; 29: 248-262.

34. Sahlstrand-Johnson P, Jannert M, Strömbeck A, Kasim KA. Computed tomography measurements of different dimensions of maxillary and frontal sinuses. BMC Med Imaging. 2011; 11: 8.

35. Quirynen M, Lefever D, Hellings $\mathrm{P}$, Jacobs R. Transient swelling of the $\mathrm{S}$ chneiderian membrane after transversal sinus augmentation: a pilot study. Clin Oral Implants Res. 2014; 25: 36-41.

36. Makary C, Menhall A, Rebaudi A. Early postoperative reactions following lateral sinus floor elevation using piezosurgery: A radiographic study. Clin Implant Dent Relat Res. 2019; 21: 888-894.

37. Makary C, Rebaudi A, Menhall A, Naaman N. Changes in Sinus Membrane Thickness After Lateral Sinus Floor Elevation: A Radiographic Study. Int J Oral Maxillofac Implants. 2016; 31: 331-337

38. Deepthi B, Shetty S, Babu C, Rohit P, Mallikarjuna D, Rai R, Mahesh L. Correlation between gingival phenotype, residual ridge height and the Schneiderian membrane. Int J Oral Implantol Clin Res. 2012; 3: 111115 . 